\title{
Lomefloxacin Hydrochloride
}

National Cancer Institute

\section{Source}

National Cancer Institute. Lomefloxacin Hydrochloride. NCI Thesaurus. Code C47587.

The hydrochloride salt form of lomefloxacin, a synthetic broad-spectrum fluoroquinolone with antibacterial activity. Lomefloxacin hydrochloride inhibits DNA gyrase, a type II topoisomerase involved in the induction or relaxation of supercoiling during DNA replication. This inhibition leads to a decrease in DNA synthesis during bacterial replication, resulting in cell growth inhibition and eventually cell lysis. 